\section{Imagery ability and visual perception of verbal and nonverbal stimuli*}

\author{
ALLAN PAIVIO and CAROLE H. ERNEST \\ University of Western Ontario, London 72, Ontario, Canada
}

Ss differing in imagery ability were required to identify letters, pictures, and geometric forms flashed one at a time to either the left or the right visual field. The three types of items were presented in homogeneous blocks, with different Ss receiving letters, pictures, or forms as their first list of items. Overall, recognition scores were higher for high imagers than for low, for pictures than for letters or forms, and in the right field than in the left. An analysis of first list data, which removed any effect of prior exposure to different types of material, yielded a significant triple interaction, showing the typical right-field superiority but no imagery effect for letters, a strong imagery effect but no field effect for pictures, and a marked left-field inferiority among low imagers in the recognition of forms. The data are discussed in terms of possible differences in functional asymmetries of the cerebral hemispheres for the two imagery groups.

This study investigated a possible perceptual basis for functional distinctions between individuals who differ in visual imagery ability. Previous studies have shown that individuals identified as high in imagery ability, according to objective tests and questionnaires, surpass low imagers in their incidental memory for visual stimuli (e.g., Ernest \& Paivio, 1969; Sheehan \& Neisser, 1969), in the speed of their imaginal and verbal associative reactions to concrete and abstract stimulus words (Ernest \& Paivio, 1971b), and in the accuracy with which they identify. stimuli exposed for a brief but suprathreshold duration (Ernest \& Paivio, 1971a).

The principal question asked in the present study was whether or not high imagery $S s$ in fact do excel in their ability to process visual material at a perceptual level and, if so, whether the ability is general or specific to certain types of stimuli. If the ability is general, it should be reflected in greater tachistoscopic recognition accuracy among high imagers regardless of the type of material being presented. On the other hand, the theoretical and operational definition of imagery in terms of figural ability suggests a relationship only between imagery ability and the recognition of nonverbal stimuli.

A distinction between the processing of verbal and nonverbal stimuli has been made in connection with the apparent functional asymmetries of man's two cerebral hemispheres (Kimura, in press). The available evidence indicates that verbal material, such as letters, are recognized

*This research was supported by grants from the National Research Council (NRC) of Canada (APA 0087) and the University of Western Ontario Research Fund to the first author and a NRC postgraduate scholarship to the second author. We thank Dr. Margaret Dumford for her advice on aspects of the procedure. better when presented to the right visual field than when presented to the left visual field. Conversely, "nonverbal" geometric forms are recognized equally well in both fields (e.g., Bryden \& Rainey, 1963; for summaries, see Kimura, in press; Paivio, 1971), and certain nonverbal stimuli are better recognized in the left field (Durnford \& Kimura, 1971; Kimura, 1969). It has been suggested that the left hemisphere, which receives visual information from the right field, is specialized for processing verbal stimuli, whereas the right hemisphere, serving the left field, appears to be specialized for certain nonverbal functions.

Given the above theory, it may be speculated that superiority on spatial or figural-transformational ability tests (i.e., high imagery ability) reflects superior right-hemispheric functioning. If true, high imagers should be more accurate than low imagers, specifically in identifying nonverbal stimuli presented to the left visual field. No imagery differences should result when such materials are presented to the right visual field nor when verbal stimuli are presented to either visual field. The following experiment explored these possibilities, using verbal and two types of nonverbal materials as stimuli in a tachistoscopic recognition task.

\section{METHOD \\ Subjects}

Seventy-seven volunteer Ss attending undergraduate psychology courses during summer school completed a test battery consisting of two spatial manipulation tasks (see Barratt, 1953) and an 87-item true-false questionnaire concerned with an individual's use of verbal and imaginal techniques in thinking and problem solving. Scores from the spatial tasks (Space Relations and the revised Minnesota Paper Form Board) and the imagery score from the questionnaire were converted to standard scores and totaled for each S. A positive total standard score defined high imagery ability, and a negative standard score defined low imagery ability.

Twenty-two high imagers (10 males and 12 females) and 17 low imagers (8 males and 9 females) were selected from the total sample. The mean standard scores for high and low imagers were +1.901 and -2.044 , respectively. Of the high imagers, 20 were right-handed and 8 wore corrective lenses; among low imagers, 15 were right-handed and 9 wore corrective lenses. ${ }^{1}$

\section{Materials}

Three types of stimuli were used: single capital letters, pictures of familiar objects, and geometric forms. Eight items were chosen within each of these stimulus attribute conditions. Only items which were approximately symmetrical in form were selected. The letters were: A, H, M, T, V, W, X, and $Y$. The pictures were selected from a pool of 80 line drawings prepared in our laboratory. They were: apple, barrel, bowl, butterfly, clock, dress, house, and vest. The geometric forms consisted of a circle, cross, diamond, rectangle, semicircle, square, star, and triangle.

Since some investigators (e.g., Bryden \& Rainey, 1963) have attributed recognition differences between pictures and geometric forms to the familiarity of these stimuli, 7-point ratings were completed by an independent group of $43 \mathrm{Ss}$ on the familiarity of the pictures and geometric forms, subsequent to this experiment. An additional 20 Ss provided data on the latency with which these stimuli elicited an overt verbal label, and the percent consistency with which 28 naive Ss gave the expected label of each stimulus was also determined. The values for familiarity $(1=$ unfamiliar; 7 = familiar), labeling latency (in seconds), consistency of labeling (in percentages), and Thorndike and Lorge (1944) frequency (where AA and $A$ words were given values of 100 and 50 , respectively) are presented in Table 1. Standard deviations are in parentheses. Comparisons by $t$ tests showed that the two types of stimuli were similar in all respects, except for mean labeling latency. Pictures were labeled more quickly than geometric forms, $t(14)=2.14, p=.05$.

The stimuli were presented in a Harvard tachistoscope (Gerbrands Model T-3B-1) at a distance of approximately $31.5 \mathrm{in}$. from S's eyes. Each stimulus was centered within a 
Table 1

Mean Values and Standard Deviations (in Parentheses) for Familiarity, Labeling Latency, Labeling Consistency, and Frequency for Pictures and Geometric Forms

\begin{tabular}{|c|c|c|c|c|}
\hline & Familiarity & $\begin{array}{c}\text { Labeling } \\
\text { Latency } \\
\text { (Sec) }\end{array}$ & $\begin{array}{c}\text { Labeling } \\
\text { Consistency }\end{array}$ & $\begin{array}{c}\text { Thorndike- } \\
\text { Lorge } \\
\text { Frequency } \\
\text { of Labels }\end{array}$ \\
\hline Pictures & $\begin{array}{c}5.71 \\
(1.03)\end{array}$ & $\begin{array}{c}2.24 \\
(0.16)\end{array}$ & $\begin{array}{c}98.62 \\
(02.50)\end{array}$ & $\begin{array}{c}\mathbf{5 3 . 1 2} \\
(29.31)\end{array}$ \\
\hline Forms & $\begin{array}{c}5.06 \\
(0.61)\end{array}$ & $\begin{array}{c}3.02 \\
(0.95)\end{array}$ & $\begin{array}{c}86.75 \\
(16.93)\end{array}$ & $\begin{array}{c}58.12 \\
(44.11)\end{array}$ \\
\hline
\end{tabular}

1 -in.-square area, $3 / 4$ in. to the right or left of center, on a $4 \times 6$ in. index card. The original pictures were photographically reduced in size until their maximum width and/or height was 1 in. The letters and geometric forms were drawn using a black ballpoint pen. The maximum width and/or height of these stimuli was similarly 1 in.

Homogeneous lists of items were constructed, with all Ss receiving all types of stimuli in one of three orders. The orders were: letters-forms-pictures (Order 1); pictures-letters-forms (Order 2); and forms-pictures-letters (Order 3). Within each stimulus attribute list, the field of presentation was randomized over trials. Each item within a list was presented twice, once in the left field and once in the right field. The field in which an item was initially located was counterbalanced across Ss. The position of items within a list was random, with the exception that at least two items intervene between identical stimuli.

\section{Procedure}

After six initial practice trials in which two instances of each type of material were presented, the experimental stimuli were presented at a constant exposure duration of $10 \mathrm{msec}$, one stimulus per trial. If $\mathrm{S}$ could not identify correctly any of the stimuli in the first list, the exposure duration was increased to $15 \mathrm{msec}$ for the repetition of that list and all subsequent lists. ${ }^{2}$ This occurred with six Ss in all. A criterion of at least one correct response per list had to be attained by each $S$ for inclusion in the experiment. Only four Ss failed to reach this criterion.

Prior to the presentation of each list, Ss were informed of the type of material to be presented and reminded to stare at the fixation point, which was exposed for $5 \mathrm{sec}$ immediately

before the presentation of each stimulus. The Ss were informed that the stimuli would appear to the left or right of the fixation point but that it was not necessary to report the side on which the item appeared. They were allowed 9.9 sec to respond orally and were encouraged to guess. Reminders to fixate were made approximately four times throughout the presentation of each list.

If a synonym for the expected label of a picture or geometric form was given, the item involved was presented under untimed conditions at the completion of that list. If $\mathrm{S}$ repeated the label previously given (e.g.: "dish" instead of "bowl"), the identification was scored as correct.

\section{RESULTS AND DISCUSSION}

An analysis of variance for unequal cell frequencies (Winer, 1962) incorporated imagery ability (high and low) and sex as between-S factors, with time (first and second time a specific item was presented within a list), stimulus attribute (letters, pictures, and geometric forms), and field (left and right) as within-S factors. Mean values for the interaction involving the three major variables are presented in Table 2 . The maximum possible score is 4 .

Significant main effects were obtained for imagery ability, $\mathrm{F}(1,35)=8.77, \quad \mathrm{p}<.01 ; \quad$ stimulus attribute, $F(2,70)=7.37, p<.01 ;$ and field, $F(1,35)=7.22, p<.05$. It is apparent from Table 2 that high imagers surpassed low imagers in mean number of correct identifications, pictures were recognized more readily than letters or geometric forms, and a right-field superiority obtained for all types of material. Recognition differences between high and low imagers tended to be smaller when the letter or verbal material was presented relative to the nonverbal material (i.e.,

Table 2

Mean Number Correct as a Function of Stimulus Type, Field, and Imagery Ability

\begin{tabular}{|c|c|c|c|c|c|c|}
\hline & \multicolumn{2}{|c|}{ Letters } & \multicolumn{2}{|c|}{ Pictures } & \multicolumn{2}{|c|}{ Forms } \\
\hline & Left & Right & Left & Right & Left & Right \\
\hline High Imagers & 1.56 & 1.91 & 1.74 & 2.16 & 1.68 & 1.73 \\
\hline Low Imagers & 1.24 & 1.27 & 1.24 & 1.32 & 0.59 & 0.94 \\
\hline
\end{tabular}

pictures and geometric forms). This interaction, however, failed to reach significance, $F(2,70)=2.63, \mathrm{p}<.10$. The triple interaction involving imagery, stimulus attribute, and field similarly fell short of significance, $F(2,70)=2.30 .3$

Because theoretical interest centered particularly on the interactions, the trends were examined further by means of separate post hoc analyses of variance for each type of stimulus, with imagery level and field as independent variables. For letters, no significant effects were obtained for imagery level, field, or their interaction, $F s(1,37) \leqslant 2.21$. For pictures, the effects were significant for imagery, $F(1,37)=9.41, p<.01$, and field, $F(1,37)=10.02, p<.01$. The interaction of imagery and field a p proached significance, $F(1,37)=3.50, p<.10$. The forms analysis yielded a highly significant effect for imagery level, $F(1,37)=16.16, p<.001$, but not for field nor its interaction with imagery, $F(1,37) \leqslant 1.63$.

The results of the post hoc analyses are consistent with the view that imagery ability is particularly relevant to the perception of nonverbal stimuli. With respect to field differences, the analyses revealed that the overall right-field effect obtained in the main analysis was primarily a function of the picture data. Bryden and Rainey (1963) and Wyke and Ettlinger (1961) have similarly found a right-field superiority for pictures of familiar objects, although Kimura (in press) has been unable to replicate this finding. Consistent with Bryden (1960) and Bryden and Rainey (1963), the present findings also suggest that geometric forms are recognized equally well in both visual fields.

The absence of a significant right-field effect for the recognition of letters, however, is not consistent with previous findings (e.g., Heron, 1957; Kimura, 1966). The inclusion of left-handed Ss in the analysis did not appear to account for this result, since these individuals demonstrated a slightly greater right-field superiority for letters than that found in the analysis. A possible explanation is suggested by Kinsbourne's (1970) attentional model of perceptual biases, in which he suggests that "if visual attention prior to stimulus exposure is biased to one side of fixation, this implies a greater readiness of the perceptual mechanism to respond to material in the attended location, with consequent superior performance [p. 195]." This attentional bias is assumed to result from differential activation of one or the other hemisphere, depending on whether verbal or nonverbal material is 


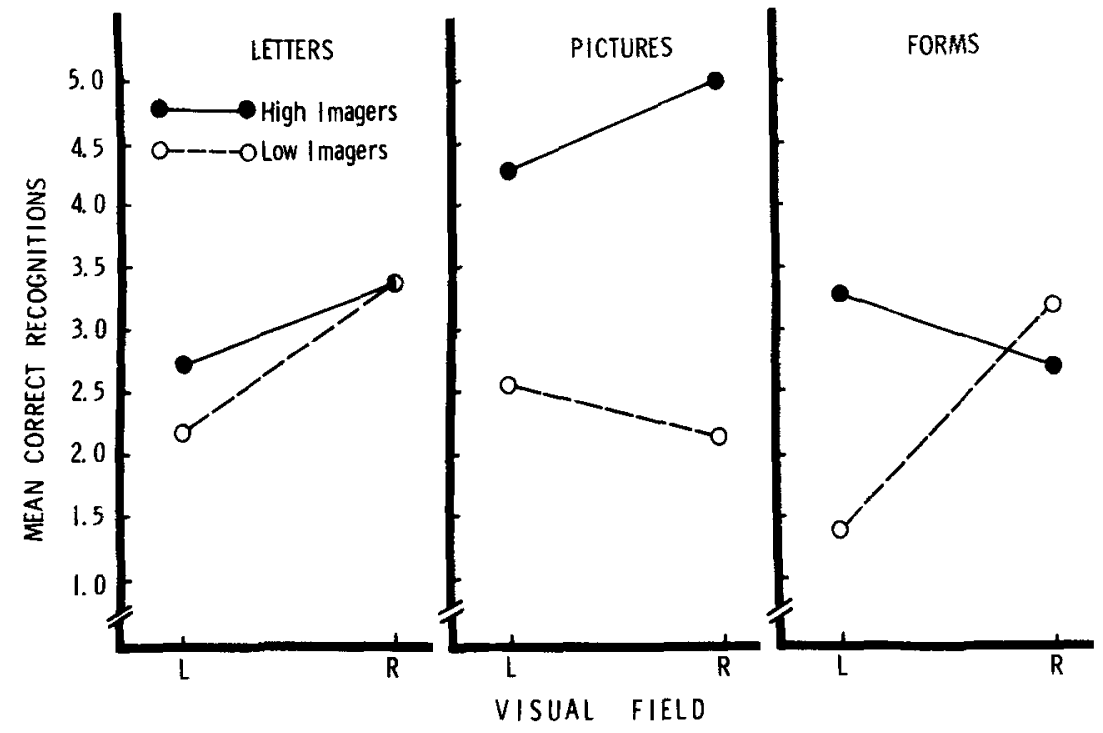

Fig. 1. First list recognition accuracy scores as a function of imagery ability, stimulus attribute, and visual field.

expected as a result of instructions or material previously experienced in the experiment. The model is relevant here if we assume that such an expectancy can be created by exposure to a list of items of a particular type in the experimental setting. Accordingly, since stimulus attribute was a within-Ss variable and list order was counterbalanced to some extent, any differential expectancy for verbal and nonverbal material would have been confounded when the data from all three lists were included in the analysis and any field differences thus obscured. Under conditions in which verbal material was not preceded by nonverbal material, a more pronounced superiority favoring the right field might emerge. Similarly, any left-field superiority for pictures and forms would be most likely to show up when these materials were not preceded by letters.

To test for such effects, an analysis of variance was carried out, using only data from the first homogeneous list presented to each $\mathrm{S}$. The independent variables were imagery ability, stimulus attribute, and field, the latter being the only within-S variable. In this analysis, the maximum possible score is 8 , since Ss' first- and second-time scores were totaled. The analysis yielded a significant main effect for field, $F(1,33)=6.19$, $\mathrm{p}<.05$, with an overall right-field superiority again being demonstrated. The effect of imagery ability fell short of significance, $F(1,33)=3.95$, $\mathrm{p}<.10$, as did the stimulus attribute effect, $F(2,33)<1$. However, imagery ability, stimulus attribute, and field interacted significantly, $F(2,33)=5.14, \quad p<.05$, thus qualifying the main effect for field.
This interaction is illustrated in Fig. 1. In order to clarify its interpretation, separate two-way analyses of variance were calculated for each stimulus attribute.

For letters, the only significant effect was for field, $F(1,11)=4.95$, $\mathrm{p}<.05$. Consistent with expectations, more letters were identified in the right visual field $(\bar{x}=3.38)$ than in the left visual field $(\bar{x}=2.54)$. Imagery ability again proved to be nonsignificant, $\mathbf{F}(1,11)<1$.

The pictures analysis yielded a significant main effect for imagery ability, $F(1,12)=11.73, p<.01$. High imagers recognized more pictures $(\bar{x}=4.64)$ than did low imagers $(\bar{x}=2.36)$. Neither the field effect nor the Imagery by Field interaction reached significance, although the latter approached significance at the .05 level, $F(1,12)=4.17$.

The only significant effect in the forms analysis involved the interaction between imagery ability and field, $F(1,10)=5.75, p<.05$. From the right portion of Fig. 1, it is evident that high imagers were slightly more accurate in the left than in the right field, whereas low imagers had considerable difficulty identifying geometric forms presented in the left field. Thus, in this case, the superiority of high imagers was specific to the left visual field.

\section{GENERAL DISCUSSION}

The results of the overall analysis indicated generally that visual recognition was easier in the right field than in the left, for pictures than for letters or geometric forms, and for high imagers than for low imagers. The superiority of pictures could be an artifact of physical rather than symbolic characteristics of the stimuli (e.g., the lines were slightly bolder in the case of pictures than in the case of words or forms) and it is of no theoretical interest in the present study. The imagery ability effect suggests a general visual-perceptual superiority among high imagery Ss, although the separate posthoc analyses of each stimulus type indicated that the effect was significant only for nonverbal material. Moreover, a somewhat different pattern resulted from the analysis of first-list data, which was intended to control for any carryover or priming effect that initial exposure to nonverbal stimuli might have on processing of letters, and vice versa. This analysis revealed only the typical right-field superiority for letters, a strong imagery effect, but no reliable field effect for pictures, and an interaction resulting mainly from marked left-field inferiority among low imagers in the recognition of geometric forms.

These findings clarify the functional nature of imagery ability in some important respects. First, imagery ability is apparently unrelated to letter recognition, but high imagers clearly surpass low imagers in picture recognition. These data suggest strongly that the superiority of high imagers is specific to nonverbal stimuli, although in themselves they do not rule out a verbal-processing component in the effect. It could be argued, for example, that high imagers may surpass low imagers in their ability to code pictures verbally and that this ability plays a part in recognition accuracy. Consistent with this view, another (unpublished) study showed that high imagers were faster than low imagers in picture naming, which can be viewed as one measure of verbal coding ability. However, the role of such an ability in picture recognition is yet to be investigated directly.

A second important feature of the results is the interaction of imagery ability and field of presentation in form recognition when such recognition is unconfounded by the effects of prior exposure to different material. Low imagers were decidedly inferior to high imagers on this task when the forms were presented in the left field, but not when they were presented in the right field. Moreover, low imagers showed a strong laterality effect favoring the right field, whereas, if anything, this was reversed in the case of high imagers. These data seem interpretable in terms of right-hemisphere functional differences between high and low imagers, although it is difficult to specify the nature of the difficulty 
experienced by the low imagers. It could be a right-hemisphere deficiency in the processing of visual information concerning forms or relative inefficiency in the transmission of appropriate information from the right hemisphere to language control systems in the left hemisphere, such information being necessary for the initiation of the verbal response that identifies the form.

Such explanations in terms of specific differences in functional asymmetries between high and low imagers must be regarded as highly speculative at this stage of factual knowledge in the problem area. This is so particularly in view of the dissimilar pattern of results for pictures and forms, which precludes any simple and consistent statement regarding perception of "nonverbal" visual patterns. Nonetheless, certain empirical conclusions appear justified: high imagers are superior to low imagers in the identification of nonverbal but not of verbal visual stimuli, and their superiority is specific to the left visual field in the case of the geometric forms, which may be regarded as the least verbal of the stimuli, at least in the sense that Ss take longer on the average to name forms than to name letters or pictures (see Morin, Konick, Troxell, \& McPherson, 1965, and the method section in the present report). Obviously, it would be desirable to investigate the problem further, using procedures in which naming is not required in the identification of nonverbal stimuli.

It should be noted, finally, that no lateral asymmetry was observed in the case of pictures when these had not been preceded by letters. The finding is consistent with Kimura (in press) but not with Bryden anci Rainey (1963). Since the latter investigators used mixed lists, it is possible that their results can be explained in terms of priming of verbal processing by the presence of letters in the lists.

\section{REFERENCES}

BARRATT, P. E. Imagery and thinking. Australian Journal of Psychology, 1953, $5,154-164$.

BRYDEN, M. P. Tachistoscopic recognition of non-alphabetic material: Canadian Journal of Psychology, 1960, 14, 78-86. BRYDEN, M. P., \& RAINEY, C. A Left-right differences in tachistoscopic recognition. Journal of Experimental Psychology, 1963, 66, 568-571.

DURNFORD、M.. \& KIMURA, D. Right-hemisphere specialization for depth perception reflected in visual field differences. Nature, 1971, in press.

ERNEST, C. H., \& PAIVIO, A. Imagery ability in paired-associate and incidental learning. Psychonomic Science, 1969, 15 181-182.

ERNEST, C. H., \& PAIVIO, A, Imagery and sex differences in incidental recall. British Journal of Psychology, 1971a,62, 67-72.

ERNEST, C. H., \& PAIVIO, A. Imagery and verbal associative latencies as a function of imagery ability. Canadian Journal of Psychology, 1971b, 25, 83-90.

HERON, W. Perception as a function of retinal locus and attention. American Journal of Psychology, 1957, 70, 38-48.

KIMURA, D. Dual functional asymmetry of the brain in visual perception Neuropsychologia, $1966,4,275-285$.

KIMURA, D. Spatial localization in left and right visual fields. Canadian Journal of Psychology, 1969, 23, 445-458.

KIMURA, D. Asymmetries in perception related to hemispheric differentiation of function. In M. Kinsbourne (Ed.), Hemispheric asymmetry of function. London: Tavistock, in press.

KINSBOURNE, $M$. The cerebral basis of
1 1:i:al asymmetrie Psychologica, 1970

ntion. Acta N., \& MCPHERSON, S. Information and reaction time for "naming" responses. Journal of Experimental Psychology, $1965,70,309-314$

PAIVIO, A. Imagery and verbal processes. New York: Holt, Rinehart, \& Winston, 1971.

SHEEHAN, P. W., \& NEISSER, U. Some variables affecting the vividness of imagery in recall. British Journal of Psychology, 1969, 60, 71-80.

THORNDIKE, E. L.. \& LORGE, I. The teacher's word book of 30,000 words. New York: Bureau of Publications, Teachers College, Columbia University, 1944.

WINER, B. J. Statistical principles in experimental design. New York: McGraw-Hill, 1962.

WYKE, M., \& ETTLINGER, G. Efficiency of recognition in left and right visual fields: Its relation to the phenomenon of visual extinction. Archives of Neurology, Chicago, 1961, 5, 659-665.

$$
\text { NOTES }
$$

1. Because of the possibility that left-handed Ss might have mixed or reversed lateralization of cerebral functions, the results of the study were analyzed both with and without the four left-handed Ss included. The pattern of results did not differ for the two analyses, and only the one for the total sample will be reported here.

2. For two Ss (one high imager and one low imager), this procedure was followed before the first list had been completely presented.

3. An analysis of variance incorporating the data from $S$ s tested at $10 \mathrm{msec}$ only yielded essentially the same results. Significant main effects were obtained for imagery ability, $F(1,29)=6.82, p<.05$ stimulus attribute, $\mathrm{F}(2,58)=7.54, \mathrm{p}<.01$; and field, $F(1,29)=12.23, p<.01$. In addition, sex differences interacted with field, $F(1,29)=4.75, p<.05$, such that males surpassed females in correctly identifying items presented to the right field (1.71 vs 1.41$)$ but not to the left field $(1,29$ vs 1.31$)$.

(Accepted for publication May 17, 1971.) 